\title{
CATACLYSMIC BINARIES: OPTICAL AND GEOMETRICAL THICKNESS OF DISKS AND THE VISIBILITY OF THE PRIMARY COMPONENTS
}

\author{
JÓZEF I. SMAK \\ N. Copernicus Astronomical Center \\ Bartycka 18 \\ 00-716 Warsaw \\ Poland
}

\begin{abstract}
Accretion disks in cataclysmic binaries are optically thick (in the vertical direction), except for the case of inner parts of cool disks in dwarf novae at quiescence, which are optically thin. In long period Algols disks are cool and optically thick; their atmospheres can be quite extensive.

Except for inclinations close to $i=0^{\circ}$, disks obscure either one or both hemispheres of the central star, the obscuration being either partial or total. In particular:

(a) In stationary accretion cataclysmic binaries (old novae and nova-like systems), at inclinations close to $90^{\circ}$ the white dwarf is fully obscured; at $i<85^{\circ}$ only its top hemisphere becomes visible.

(b) In dwarf novae at quiescence, at inclinations close to $90^{\circ}$, the obscuration of the white dwarf is either complete (for disk radii $R_{\mathrm{d}} \geq 4 \times 10^{10} \mathrm{~cm}$ ), or limited to its equatorial parts (for $\left.R_{\mathrm{d}} \leq 2 \times 10^{10} \mathrm{~cm}\right)$; at $i \approx 85-88^{\circ}$ the top hemisphere becomes fully visible, while the bottom hemisphere is fully obscured; at $i<85^{\circ}$, due to the low optical thickness of the inner disk, parts of the bottom hemisphere near the equator also become visible.
\end{abstract}

(c) In long period Algols the situations is similar as under (a); in addition, depending on inclination, part of the obscuration can be due to the atmosphere of the disk.

\section{Introduction}

It is commonly recognized that accretion disks in binary systems contribute significantly to their observed, integrated fluxes and spectra. Disk eclipses have been extensively studied in cataclysmic variables, as well as in other types of binaries. In some cases it has also been noted that the central star (the primary component) is likely to be obscured by the accretion disk, but the significance of this effect appears to be not quite fully appreciated. The purpose of the present paper is to study this problem in a more systematic way.

\section{Models of the Vertical Structure}

Crucial for the problem is the optical and geometrical thickness of the disk. With this in mind, equations of the vertical structure of an accretion disk were integrated for a wide range of the parameters involved (the basic assumptions, procedures, and details were the same as in Smak 1984; for references to other papers on the vertical structure $c f$. recent review by Meyer-Hofmeister and Ritter 1991). Is is worth to emphasize here that in the optically 
thin case models of this type must be considered as very crude. It may be comforting to note, however, that the use of the Rosseland mean opacities appears to give a reasonably good approximation (Tylenda 1988).

The problem involves four free parameters: the mass of the central star $M_{*}$, the radial distance $R$, the effective temperature $T_{\mathrm{e}}(R)$, and the viscosity parameter $\alpha$. The mass of the central star was put $M_{*}=1 M_{\odot}$; note that $M_{*}$ enters only via the combination $G M_{*} / R^{3}$ and hence the results can be rescaled for other values of $M_{*}$. Concerning the value of $\alpha$, we may recall that the observed time scales of dwarf nova outbursts imply - for the hot branch of the $\Sigma-T_{\mathrm{e}}$ relation $-\alpha \approx 0.2$ (cf. Smak 1984); on the other hand, models of dwarf nova outbursts require that $\alpha$ on the cool branch be about 4 times lower (cf. references in Meyer-Hofmeister and Ritter 1991), i.e. $\alpha \approx 0.05$. Accordingly calculations were made for a range of $\alpha=0.03-0.3$ with $\alpha=0.1$ being the standard, reference value.

Fig.1 shows the results of model calculations, with $\alpha=0.1$, in the familiar $\Sigma-T_{e}$ diagram. The lines of $z_{\mathrm{o}} / R=$ const. indicate that at large radii, even at low temperatures, disks become geometrically thick. If follows than that, unless the value of $\alpha$ is significantly larger than 0.1 , geometrically thin disk models may be not applicable for the very long period binaries (e.g. symbiotics).

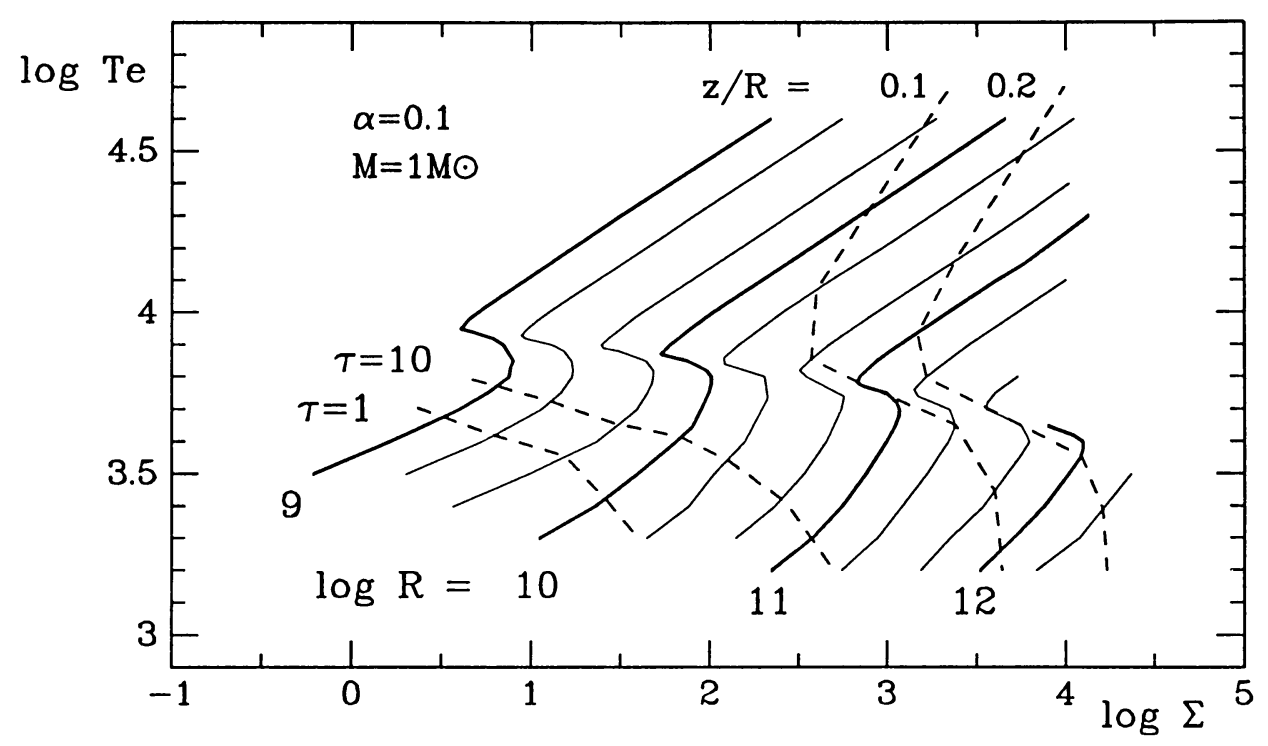

Figure 1. $\Sigma-T_{\mathrm{e}}$ relations for $\log R=9.0,9.3,9.7,10.0, \ldots, 12.3$. Also shown are the lines of $\tau=1$ and 10 and $z / R=0.1$ and 0.2 .

We now turn to the optical thickness. From the location of the $\tau=$ const. lines we confirm what has been found earlier by many authors that, generally, accretion disks in binary systems are optically thick. The only exception is the region of low temperatures and small radii. This is the case encountered in dwarf novae at quiescence. The results obtained for Z Cha by Wood et al. (1986) and for OY Car by Wood et al. (1989) show that the 
surface brightness temperatures of disks in these two systems are about $T \approx 4000-5000^{\circ}$, with only weak dependence on $R$. Adopting $T=4000^{\circ}$ we get from Fig. 1 that $\tau=1$ occurs at $R \approx 3 \times 10^{9} \mathrm{~cm}$ while $\tau=10$ - at $R \approx 1 \times 10^{10} \mathrm{~cm}$. If we now note that $(a)$ the temperatures of the inner parts are somewhat higher than $4000^{\circ}$ and (b) the value of $\alpha$ for the cool branch is very likely lower than 0.1 , then we can conclude that our values of $R$ are actually the upper limits. The overall conclusion then is that in dwarf novae at quiescence only the inner parts of disks, with $R<1 \times 10^{10} \mathrm{~cm}$, are optically thin.

The geometrical thickness $z_{0}$ in the vertical direction and, consequently, $z_{0} / R$ can be defined at two levels: at $\rho=10^{-10} \mathrm{~g} / \mathrm{cm}^{3}$, and at $\tau=1$ (provided the optical thickness $\tau_{\mathrm{o}}$ is larger than 1). The results can be represented by the following approximate formula

$$
\log \left(\frac{z_{\mathrm{o}}}{R}\right) \approx c_{\mathrm{o}}+c_{\mathrm{T}} \log T_{\mathrm{e}}+c_{\mathrm{R}} \log R-\frac{1}{3} c_{\mathrm{R}} \log \left(M_{*} / M_{\odot}\right)+c_{\alpha} \log (\alpha / 0.1),
$$

with coefficients $c_{\mathrm{o}}, c_{\mathrm{T}}, c_{\mathrm{R}}$, and $c_{\alpha}$ for the four different cases being listed in Table 1 . We note that, with the exception of case 4 , the dependence on $\alpha$ is rather weak. The shape of the disk can be approximated by $z_{0} / R \sim R^{\beta}$. In the stationary accretion case we have $\beta$ in the range 0.04-0.2 and, hence, the disk is slightly concave. In the case of dwarf novae at quiescence, when $T_{\mathrm{e}} \approx$ const. (cf. above) we have, for case 2 of Table $1, \beta=c_{\mathrm{R}} \approx 0.5$ and, hence, the disk is strongly concave.

TABLE 1

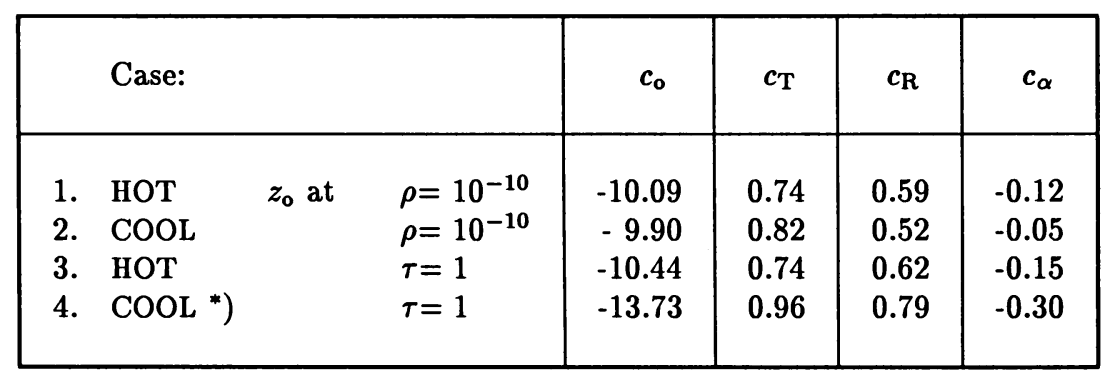

*) only for $R \geq 10^{11} \mathrm{~cm}$.

\section{Visibility of the Primary Component}

\subsection{OPTICALLY THICK DISK}

The degree of obscuration of the central star by the disk, which is generally different for the star's top and bottom hemispheres, depends on the following three parameters: the inclination $i$, the ratio $R_{\mathrm{d}} / R_{*}$, and $z_{\mathrm{o}} / R$ at $R=R_{\mathrm{d}}$, where $R_{\mathrm{d}}$ is the outer radius of the disk. Note that we implicitly assume here that the disk is concave and optically thick in the radial direction. Let us define the visible fraction of either the top or the bottom hemisphere 


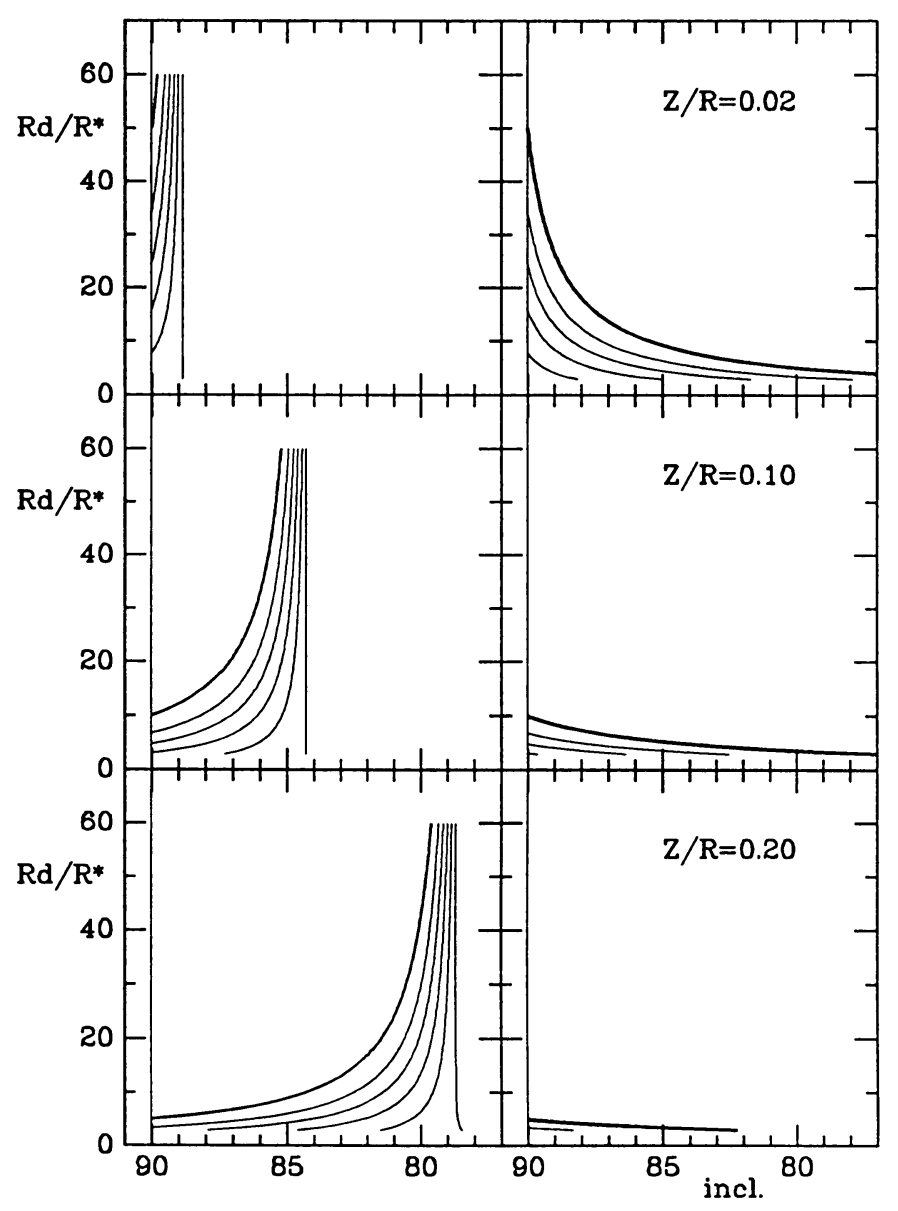

Figure 2. Equal obscuration lines, $f_{\mathrm{T}}=$ const. and $f_{\mathrm{B}}=$ const., in the $R_{\mathrm{d}} / R_{*}$ versus $i$ diagrams for three values of $z_{0} / R$. The left panel is for the top hemisphere. The right panel is for the bottom hemisphere. For the top hemipshere the area of full obscuration lies above and to the left of the thick lines corresponding to $f_{\mathrm{T}}=0$. Likewise, for the bottom hemipshere the area of full obscuration lies above the thick lines corresponding to $f_{\mathrm{B}}=0$. Thin lines are for $f=0.1,0.2,0.3,0.4$, and 0.5 .

as $f=\left(\right.$ visible area) $/ \pi R_{*}^{2}$, so that without obscuration $f_{\mathrm{T}}+f_{\mathrm{B}}=1$, and for $i<90^{\circ}$ we have $f_{\mathrm{T}}>0.5$ and $f_{\mathrm{B}}<0.5$. Calculations of $f_{\mathrm{T}}$ and $f_{\mathrm{B}}$ were made for a broad range of parameters. The results, for three representative values of $z_{\mathrm{o}} / R$, are shown in Fig.2 where equal obscuration lines are plotted in the $R_{\mathrm{d}} / R_{*}$ versus $i$ diagrams. 


\subsection{COOL, OPTICALLY THIN DISK}

The approach used in Section 3.1 cannot be applied to the case of disks in dwarf novae at quiescence, since their inner parts are optically thin. To estimate the visibility of the central star in this case we adopt a simple model of an isothermal disk. Such an approximation is suggested by two facts: (1) $T(z) \approx$ const. when the optical thickness is low (cf. Canizzo and Wheeler 1984, Meyer and Meyer-Hofmeister 1984); (2) in dwarf nova disks at quiescence $T(R)$ varies only slightly with $R$ (Wood et al. 1986,1989). It should be emphasized that at larger radii, where the disk becomes optically thick, this approximation leads to an underestimate of temperatures. Consequently, at low temperatures where the opacity increases with $T$, it will also lead to an underestimate of the optical thickness.

From the equation of hydrostatic equilibrium we have

$$
\rho=\rho_{c} \exp \left[-\left(\frac{z}{z_{0}}\right)^{2}\right],
$$

where

$$
\rho_{c}=\frac{\Sigma}{z_{\mathrm{o}} \sqrt{\pi}}
$$

and

$$
z_{\mathrm{o}}=\left(\frac{2 \frac{k}{\mu H} T}{G M_{*} / R^{3}}\right)^{1 / 2}
$$

For the surface density in Eq.(3) we can use $\Sigma=\Sigma(R, T)$ resulting from models described in Section 2.

Using the density distribution given by Eq.(2) we can now calculate the optical thickness in projection along the line of sight, as a function of the projected $z$-distance (up and down) from the center of star. For a given $T$ we are left with only two free parameters: the radius of the disk $R_{\mathrm{d}}$ and the inclination $i$. The calculations were made with $M=1 M_{\odot}$ and $T=4000^{\circ}$ (cf. Section 2). The results are shown in Fig.3.

\subsection{EXTENDED ATMOSPHERE}

Outer parts of large, cool disks, with low effective gravities, have fairly extended atmospheres. Assuming, for simplicity, that the atmosphere is isothermal we can write

$$
\rho=\rho_{\tau=1} \exp \left[-\left(\frac{\Delta z}{\Delta z_{\mathrm{o}}}\right)^{2}\right],
$$

where $\Delta z=z-z_{\tau=1}$ and $\Delta z_{\mathrm{o}}$, given by the same expression as $z_{\mathrm{o}}$ in Eq.(4), is now the characteristic scale-height of the atmosphere. 


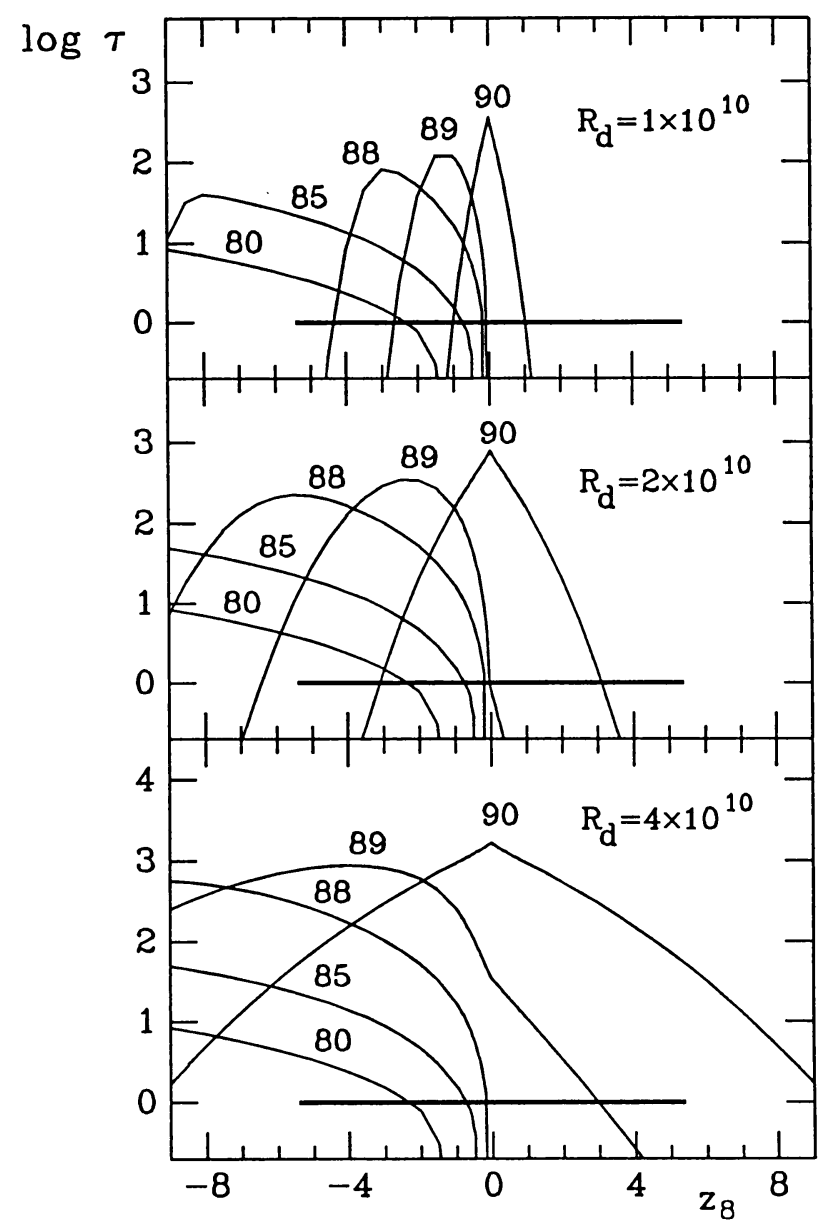

Figure 3. Optical thickness in projection along the line of sight, as a function of the inclination and the radius of the disk. $z_{8}$ is the projected $z$-distance in units of $10^{8} \mathrm{~cm}$. Thick lines at $\log \tau=0$ show the diameter of a $1 M_{\odot}$ white dwarf.

\section{Discussion}

\subsection{STATIONARY ACCRETION CATACLYSMIC BINARIES}

Typical values of parameters in the case of stationary accretion CV's (i.e. in novae and nova-like systems) are: $M=1 M_{\odot}, R_{\mathrm{d}}=5 \times 10^{10} \mathrm{~cm}$, and $T_{\mathrm{e}}\left(R_{\mathrm{d}}\right) \approx 1 \times 10^{4}$. These give: $R_{\mathrm{d}} / R_{*}=100$ and $z_{\mathrm{o}} / R \approx 0.1$ (or somewhat larger). With these values we find from Fig.2 that the bottom hemisphere is always fully obscured. The visibility of the top hemisphere depends on inclination: close to $90^{\circ}$ it is also fully obscured and becomes visible only at $i<85^{\circ}$ (or even at lower inclinations if $z_{\mathrm{o}} / R>0.1$ ). 


\subsection{DWARF NOVAE AT QUIESCENCE}

Typical values of parameters in this case are: $M=1 M_{\odot}, T_{\mathrm{e}} \approx 4 \times 10^{3}$, and $R_{\mathrm{d}}=1-2 \times$ $10^{10} \mathrm{~cm}$ for ultra-short period dwarf novae (Case $a$ ) or $R_{\mathrm{d}} \approx=4 \times 10^{10} \mathrm{~cm}$ for dwarf novae with $P>3$ hours (Case $b$ ). These give: $z_{\mathrm{o}} / R \approx 0.02$ (or slightly larger) and $R_{\mathrm{d}} / R_{*}=20-40$ (Case $a$ ) or $R_{\mathrm{d}} / R_{*} \approx 80$ (Case $b$ ). In the optically thick approximation (Section 3.1 and Fig.2) our conclusions would be as follows: at inclinations close to $90^{\circ}$ the white dwarf is either partly (Case $a$ ) or fully (Case b) obscured; in the former case the obscuration being limited to the equatorial belt. At inclinations lower than about $i=88-89^{\circ}$ the top hemisphere should become fully visible, while the bottom hemisphere - fully obscured. These conclusions undergo some modifications when we use the more realistic model of Section 3.2. From Fig.3 we find that the top hemisphere is fully visible and the bottom hemisphere fully obscured only for $i \approx 85-88^{\circ}$; at lower inclinations, due to the low optical thickness of the inner parts of the disk, the equatorial parts of the bottom hemisphere also become visible.

\subsection{LONG PERIOD ALGOLS}

As an example we consider RZ Oph (cf. Olson 1987, Zola 1991, and other references therein). We have: $M_{*} \approx 5 M_{\odot}, R_{*} \approx 4 R_{\odot}, R_{\mathrm{d}} \approx 90 R_{\odot}$, and $T_{\mathrm{e}}=4000-5000^{\circ}$ which give: $R_{\mathrm{d}} / R_{*}=20-25$ and $z_{\mathrm{o}} / R$ larger than 0.2 . From Fig.2 we find that for inclinations larger than $80^{\circ}$ the star is fully obscured and that only the top hemisphere becomes visible at lower inclinations. We also find that the characteristic scale height in the atmosphere (cf. Section 3.3) is comparable, or even larger than the radius of the star. Hence there is a range of intermediate inclinations at which the top hemisphere is obscured by the disk atmosphere. The existence of such an effect is confirmed by, and provides a natural explanation for, the observed F5Ib spectral type of the $5 M_{\odot}$ main sequence primary in RZ $\mathrm{Oph}$.

\subsection{CONCLUSIONS}

The main conclusion is that the obscuration of the primary component by the accretion disk is a common and very important effect which occurs in nearly all situations, encountered in cataclysmic variables and in other types of binaries. Consequently, it should be properly taken into account in all types of studies dealing with the analysis of the eclipse light curves, derivation of luminosities and colors of the primary, etc. It should also be emphasized that in situations involving optically thin parts of disks the obscuration effect is wavelength dependent.

\section{References}

Canizzo, J.K., Wheeler, J.C. 1984 Ap.J.Suppl., 55, 367.

Meyer, F., Meyer-Hofmeister, E. 1984 Astron.Astrophys., 132, 143.

Meyer-Hofmeister, E., Ritter, H. 1991 In: Close Binaries, J. Sahade, Y. Kondo, G. McCluskey (eds.), Kluwer Academic Publ., in press. 
Olson, E.C. 1987 Astron.J., 94, 1309.

Smak, J. 1984 Acta Astron., 34, 161.

Tylenda, R. 1988 Astron.Astrophys., 197, 225.

Wood, J., Horne, K., Berriman, G., Wade, R., O’Donoghue, D., Warner, B. 1986 M.N.R.A.S., $219,629$.

Wood, J., Horne, K., Berriman, G., Wade, R., 1989 Ap.J., 341, 974.

Zoła, S. 1991 Acta Astron., 41, in press. 